\title{
Personality Openness Predicts Driver Trust in Automated Driving
}

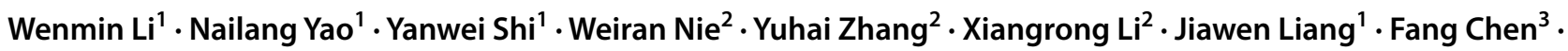 \\ Zaifeng Gao' ${ }^{1}$
}

Received: 10 May 2019 / Accepted: 18 December 2019 / Published online: 21 February 2020

(c) The Author(s) 2020

\begin{abstract}
Maintaining an appropriate level of trust in automated driving (AD) is critical to safe driving. However, few studies have explored factors affecting trust in AD in general, and no study, as far as is known, has directly investigated whether driver personality influences driver trust in an $\mathrm{AD}$ system. The current study investigates the relation between driver personality and driver trust in $\mathrm{AD}$, focusing on Level $2 \mathrm{AD}$. Participants were required to perform a period of $\mathrm{AD}$ in a driving simulator, during which their gaze and driving behavior were recorded, as well as their subjective trust scores after driving. In three distinct measures, a significant correlation between Openness and driver trust in the AD system is found: participants with higher Openness traits tend to have less trust in the AD system. No significant correlations between driver trust in AD and other personality traits are found. The findings suggest that driver personality has an impact on driver trust in AD. Theoretical and practical implications of this finding are discussed.
\end{abstract}

Keywords Automated driving $\cdot$ Trust $\cdot$ Personality $\cdot$ Openness

$\begin{array}{ll}\text { Abbreviations } \\ \text { ACC } & \text { Adaptive cruise control } \\ \text { AD } & \text { Automated driving } \\ \text { AOI } & \text { Areas of interest } \\ \text { DOF } & \text { Degree of freedom } \\ \text { HMI } & \text { Human-machine interface } \\ \text { L2 } & \text { Level 2 } \\ \text { NDRT } & \text { Non-driving-related task }\end{array}$

Wenmin Li, Nailang Yao, Yanwei Shi have contributed equally to this work.

\section{Fang Chen}

fang.chen@chalmers.se

Zaifeng Gao

zaifengg@zju.edu.cn

1 Department of Psychology and Behavioral Sciences, Zhejiang University, Hangzhou, China

2 Huawei Technologies Co., Ltd, Shenzhen, China

3 Chalmers University of Technology, Göteborg, Sweden

\section{Introduction}

Automated driving (AD) in commercially available vehicles is growing rapidly and the advances of AD technology potentially lead to a safer and more efficient traffic environment. However, challenges remain in the area of human factors. One of the most important issues is driver trust in $\mathrm{AD}$ vehicle systems. The concept of trust originally addressed interpersonal trust, but it has been extended to the domain of automation and vehicles, where trust is defined as "the attitude that an agent will help achieve an individual's goal in a situation characterized by uncertainty and vulnerability" [1]. Trust in automation has been the focus of substantial research in the past decade. Trust has been identified as a crucial factor influencing the acceptance and the use of automated technology in general [1-7], and it is now regarded as a prominent human-machine interface (HMI) issue in $\mathrm{AD}$ in particular. Building an appropriate level of trust in automation is critical to improving the productivity and ensuring the safety of human-automation pairs. Accidents could occur when drivers misuse AD due to under-trust or over-trust [6]. Indeed, excessive trust in the AD system was one key factor leading to a fatal $\mathrm{AD}$ traffic accident and two drunk driving cases involving AD in 2018 [8-10].

To date, several studies have been conducted to explore the factors influencing driver trust in AD. These studies 
largely fall into three categories according to the threelayered framework of trust proposed by Hoff and Bashir [11]. In this framework, trust is influenced by three distinct sources: the person who trusts, the system to be trusted and the situation. The source of person refers to an individual's overall tendency to trust automation. It relates to individual factors such as gender, age, culture background and personality. Researchers examined the influence of gender and age on $\mathrm{AD}$ trust $[12,13]$ and found that older adults were more likely to trust $\mathrm{AD}$, yet no difference was found between male and female operators. The source of system represents a person's knowledge of a system drawn from past experience or interaction with the system. It relates to the automated system, including factors such as system reliability, transparency and performance. Currently two lines of $\mathrm{AD}$ research have examined this aspect. The first line addressed the effect of system transparency on AD trust, where transparency was enhanced by, for instance, visually displaying uncertainty information of the system [14], offering the status of adaptive cruise control (ACC) [15] and explaining the actions of the $\mathrm{AD}$ system to drivers [16]. Consistently, researchers found that enhancing $\mathrm{AD}$ system transparency considerably raised levels of driver trust. The second line examined the role of social cues and revealed that anthropomorphism (including human-like voice, appearance and gender) $[17,18]$ and sharing the goals between the AD system and the driver [15] were associated with the increasing trust in AD. The source of situation includes both the external environment (e.g., system complexity and workload) and internal context-dependent characteristics of the operator (e.g., mood, knowledge relevant to the automated system and attentional capacity of the operator). The existing $\mathrm{AD}$ studies have focused predominantly on internal characteristics of the operator. Researchers consistently demonstrated that take-over experience of an AD system allowed a driver to recognize the shortcomings of the system, resulting in better understanding of the system, hence increasing trust level $[12,13,19]$. Payre et al. [20] further showed that extensively training drivers (e.g., in overtaking a car or being overtaken by a car) could reduce the negative impact of over-trust. Meanwhile, Molnar et al. [13] noticed that a driver's trust in the AD system was also correlated with his/her comfort with other drivers.

Although Szalma and Taylor [21] emphasized the importance of incorporating individual differences into automation design by identifying relevant personal characteristics, no study yet, as far as is known, has investigated how personality factors relate to driver trust in AD. Personality reflects an individual's stable cognition, behavior and emotional patterns that evolve from biological and environmental factors. An individual's personality is generally stable over time, and about half of the variance is rooted in one's genes rather than in the external environment. Therefore, personality has served as one of the key factors in studies of individual differences. There is growing recognition that trust in automation may be influenced by operator personality [22, 23]. For instance, using the Big Five Inventory to measure personality, Chien et al. [24] found that the Agreeableness trait and Conscientiousness trait were positively correlated to an individual's initial trust in automation in general. McBride et al. [25] explored the relation between personality and acceptance of automated decision aids by using the Myers-Briggstype indicator (MBTI) to measure personality. They found that personality traits had a significant impact on the acceptance of automated decision aids. While the previous studies shed light on the relation between personality and trust in general automation, an AD system is special since it operates in a more complex and riskier environment, so direct evidence is required to further understand the relation. The current study attempts to close this gap by examining the relation between personality and $\mathrm{AD}$ trust.

There are different methods to measure an individual's personality, and the five-factor model [26] is one of the most widely adopted approaches to personality description [27]. This model claims that five distinct traits constitute human personality: Extraversion, Agreeableness, Conscientiousness, Neuroticism and Openness. Extraversion mainly addresses preferences for social interaction, but it also includes characteristics of activity level, assertiveness, preference for excitement and stimulation as well as positive affect [26]. Individuals with high Extraversion are more likely to enjoy environments that offer opportunities for interaction and stimulation. Therefore, Extraversion might positively correlate with $\mathrm{AD}$ trust because a driver has more freedom to interact with other media or equipment in an AD context. Agreeableness is an interpersonal trait which is related to characteristics such as the propensity to trust others, helpfulness, kindness and sympathy [26]. Individuals high in Agreeableness usually adapt well to interpersonal settings involving cooperation and social interaction [28-30]. Because the AD system can be considered as a cooperative agent in achieving the goal of safe driving, it is predicted that drivers with high Agreeableness might trust the AD system more. This prediction is partially supported by the significant positive correlation between Agreeableness and initial trust in general automation, as found by Chien et al. [24]. Conscientiousness primarily addresses whether an individual is well organized or reliable in a task [26]. Notably, Chien et al. [24] also detected a significant positive correlation between Conscientiousness and people's initial trust in general automation; thus, a similar relation between Conscientiousness and AD trust might exist. Neuroticism measures an individual's typical level of emotional stability [26]. Individuals high in this trait are more easily anxious and irritable when facing tasks that include uncertainty regarding the occurrence of events and threat stimuli 
[27]. In level 2 and $3 \mathrm{AD}$, the driver is still required to take over vehicle control in the short term when an unexpected event happens, so a negative correlation between Neuroticism and AD trust is anticipated. Finally, Openness captures a preference for such traits as independent judgment, enjoyment of variety and novelty, being curious and seeking new experiences, active imagination and aesthetic sensitivity [26]. Open individuals may enjoy intellectually challenging activities, because Ackerman and Heggestad [31] found Openness positively correlated with an individual's typical level of intellectual engagement in activities. If a task situation met this profile, higher Openness trait might correlate with better performance and higher trust. However, one goal of $\mathrm{AD}$ systems is to free drivers from the concentration and cognitive load of driving, essentially removing the "intellectually challenging activities" that are part of manual driving. Therefore, a negative correlation is expected between Openness trait and AD trust.

In the current study, the participants were required to first complete a personality questionnaire, then conduct a Level 2 (L2, also named Partial Driving Automation by SAE [32]) $\mathrm{AD}$ for about $40 \mathrm{~min}$ in a simulator, and finally answer a trust questionnaire. To examine driver trust in the AD system and clarify the relation between personality and trust, drivers were required to engage in a non-driving-related task (NDRT) during the AD. Specifically, drivers conducted a mathematical addition task on a tablet when the car was in the automatic mode. Critically, the drivers were informed that accidents might occur (e.g., hitting a pedestrian) due to the limitation of the AD system, and they were instructed to take over the vehicle as soon as possible whenever they thought the system was no longer able to support safe driving. Correlations between the personality dimensions of the five-factor model and the measure of AD trust were examined.

\section{Method}

\subsection{Participants}

Thirty-two students from Zhejiang University were recruited to participate in the study. Two participants were excluded from analysis due to failure to understand the task; two participants were excluded from analysis because complete eye-tracking data could not be recorded with sufficient quality (i.e., with a detectable pupil in at least $80 \%$ of frames). Overall, there were 28 valid participants (14 males), with an average age of 23.3 years old $(\mathrm{SD}=1.4)$. All participants had normal or corrected-to-normal vision acuity and no physical disabilities. They all had a driver's license, for an average of 2.3 years $(S D=1.6)$. All the

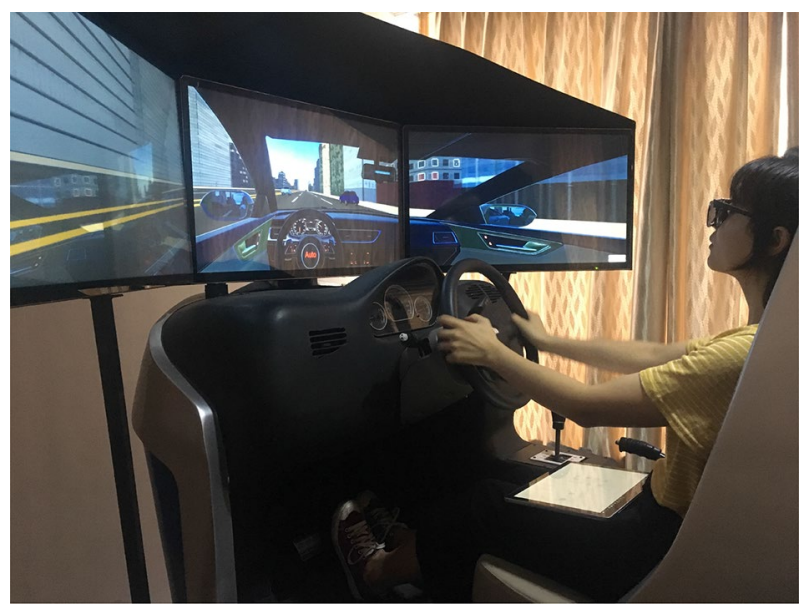

Fig. 1 Setup of the driving simulator

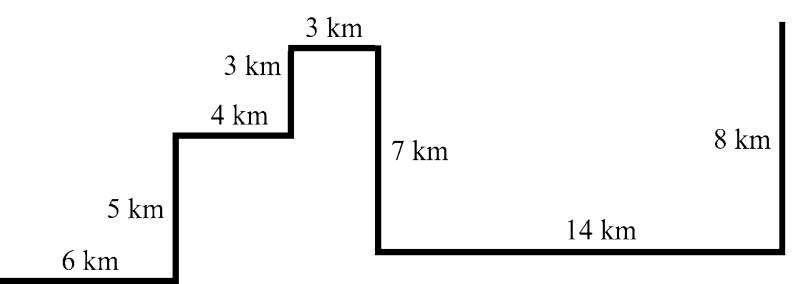

Fig. 2 Aerial view of the driving route

participants had no AD experience (including an AD simulator) prior to this study. The participants received 40-50 RMB after the experiment.

\subsection{Apparatus}

The study was conducted in a driving simulator. The simulator was mounted on a 6 degrees of freedom (DOF) hexapod motion platform, allowing a displacement of $\pm 23.5 \mathrm{~cm}$ and a rotation of $\pm 30^{\circ}$. Eye-tracking data were recorded using head mounted Tobii Pro Glasses 2 with a sampling rate of $50 \mathrm{~Hz}$. The driving behavioral data were recorded using RealSense $\left(\right.$ Intel ${ }^{\circledR}$ ), which was set at the left side of the simulator. A 12.3-inch Surface Pro 5 tablet was used to conduct the NDRT.

The driving environment was presented via three LED monitors (resolution: $1920 \times 1080$ pixels; refresh rate: $60 \mathrm{~Hz}$ ), with an angle of $150^{\circ}$ between them (see Fig. 1). The driving task was programmed using the Unity $3 \mathrm{D}$ engine to simulate a city driving environment. An aerial view of the driving route is shown in Fig. 2; all the corners were intersections. The total driving distance was $50 \mathrm{~km}$. To simulate city driving, the average autonomous driving speed was maintained at $60-70 \mathrm{~km} / \mathrm{h}$. 
To simulate real situations in $\mathrm{L} 2 \mathrm{AD}$, three categories of emergent events were set where accidents could occur if the driver did not respond in time:

(a) Facing a traffic accident: while the AD ran smoothly in the middle lane, suddenly there was a car accident ahead. The AD system would change the lane at a distance of $60 \mathrm{~m}$ and go back to the middle lane after $20 \mathrm{~m}$ away from the accidents (see Fig. 3a). However, the AD system might also fail to change lanes and lead to an accident. (b) Urgent braking: while the AD ran smoothly in the middle lane, a car in the left lane overtook the AD vehicle and changed to the middle lane, but then braked sharply ahead of the AD vehicle (see Fig. 3b). The AD system started to decelerate at a distance of $40 \mathrm{~m}$ away from the car ahead. However, it might be too late to decelerate, leading to an accident.

(c) Pedestrians crossing the road: while the AD ran smoothly in the middle lane, a group of pedestrians started crossing the road ahead (see Fig. 3c). The AD system started to decelerate at a distance of $40 \mathrm{~m}$ away a

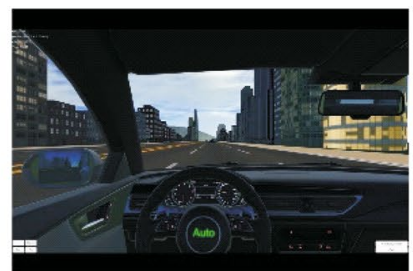

Automated mode

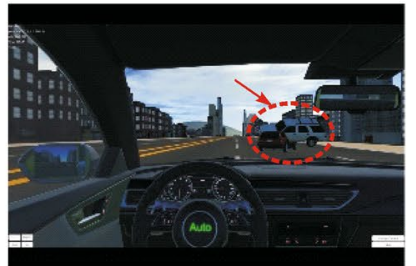

Changing lanes*

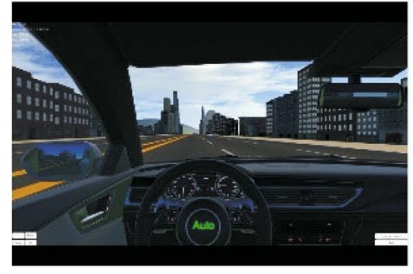

Backing to the middle lane

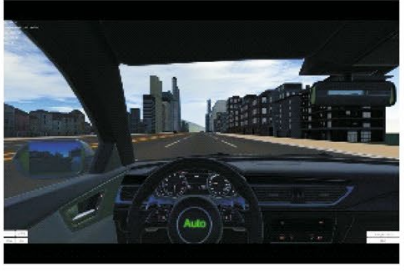

Automated mode

*If there is a car passing by on the left side at this time,the system will stop to wait for the left car to pass safely and then change lanes

b

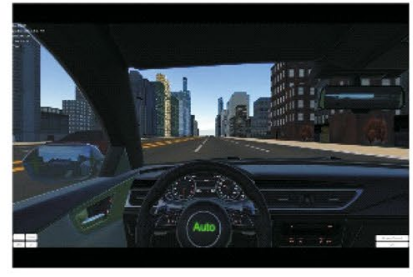

Automated mode

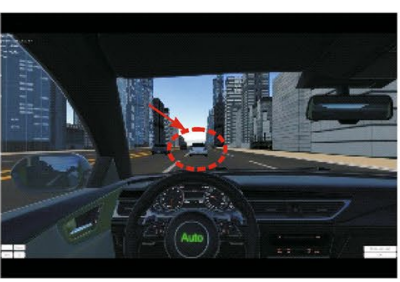

The front car

changes lanes

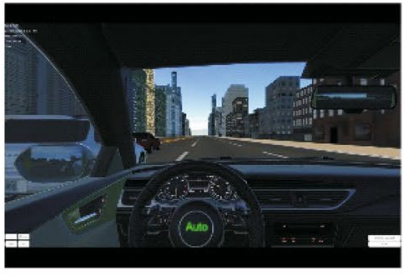

A car overtakes from the left side

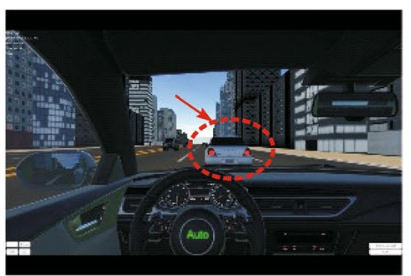

When the front car brakes sharply*,the system starts to decelerate $40 \mathrm{~m}$ away

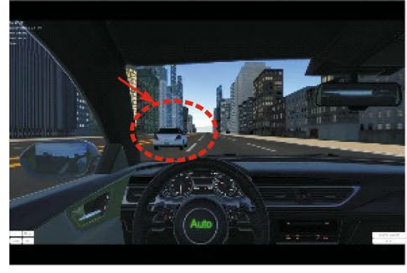

The front car tends to change lanes

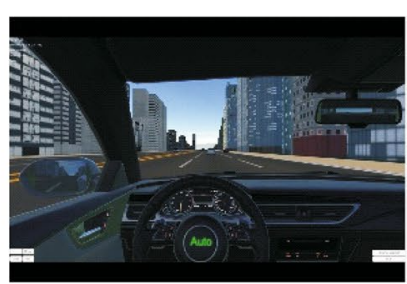

Two cars gradually adjust to safety distance

*The system is designed to stop safely within $40 \mathrm{~m}$ when the car is driving at a maximum speed of $70 \mathrm{~km} / \mathrm{h}$. Thus the deceleration should be $4.72 \mathrm{~m} / \mathrm{s}^{2}$

C

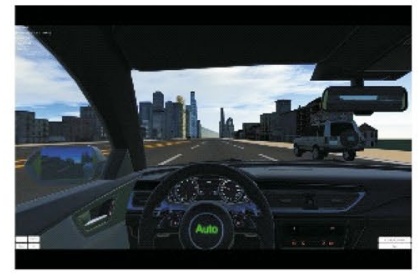

Automated mode

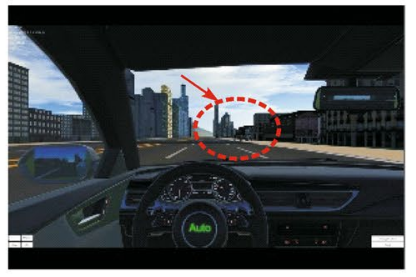

Pedestrians cross the road*

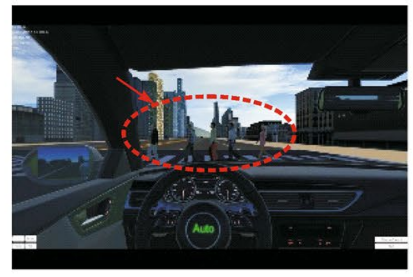

The car waits until all pedestrians to cross the road

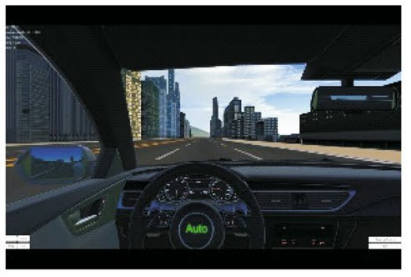

The car restarts

${ }^{*}$ If there is a car in front of the zebra crossing,the system will start to decelerate when it is $40 \mathrm{~m}$ away from the front car.

Fig. 3 Emergency scenarios: a Facing a traffic accident, b Urgent braking, $\mathbf{c}$ Pedestrians crossing road. The dashed red circles were used to indicate the key elements in the three emergent events 
from the pedestrian crossing and stopped before the crossing until all pedestrians had crossed the road. However, the AD system might fail to stop before the crossing and hit a pedestrian.

\subsection{Measurements}

Driver trust in the AD system was measured through both objective and subjective measurements. For the objective measurements, driving behavior and gaze behavior were adopted as the indices. Participants' responses to the trust questionnaire were used for the subjective measurement.

\subsubsection{Questionnaire}

2.3.1.1 Personality Driver personality traits were measured by the Chinese Big Five Personality Inventory Brief (CBF-PI-B, [33-35]). There were 40 items in the scale, each measured on a 6-point Likert scale $(1=$ totally disagree, $6=$ totally agree), with 8 items for each dimension. The total score of the 8 questions in each dimension was calculated as the score of the corresponding trait.

2.3.1.2 Trust Driver trust in the AD system was measured using a modified trust questionnaire [15] of Jian et al. [36]. It consists of 7 questions, each measured on a 7-point Likert scale ( $1=$ totally disagree, $7=$ totally agree $)$, and it is well-accepted in automation trust studies. The average score indicates the trust level, with higher scores indicating higher levels of trust.

The data from both questionnaires are at an interval level [24, 37-39].

\subsubsection{Gaze Behavior}

Driver gaze attention to the driving related areas during driving reflects the trust level. Specifically, the higher the driver's trust of the AD system, the less frequently the driver monitors the system $[12,19]$.

In the current study, the areas of interest (AOI) were defined as the windshield, mirrors, side windows and steering wheel. According to the default setting of Tobii Pro Glasses, two or more glances to the AOI separated by blinks less than $75 \mathrm{~ms}$ were combined, while glances shorter than $60 \mathrm{~ms}$ when passing through AOI were eliminated from analysis.

In line with Hergeth et al. [19], monitoring frequency and ratio of AOI served as the key indices. Monitoring frequency was calculated as the number of monitoring glances to the AOI during the NDRTs scaled to the duration of NDRTs: Monitoring frequency $=$ AOI fixation count/duration of NDRTs. Monitoring ratio was calculated as the time a driver spent on monitoring the AOI scaled to the duration of NDRTs: Monitoring ratio = duration of AOI total fixation/ duration of NDRTs.

\subsubsection{Driving Behavior}

It has been suggested that take-over behavior during $\mathrm{AD}$ is tightly related to trust. For instance, the more trust in the $\mathrm{AD}$ system, the longer a driver waits before taking over the system [13]. Take-over rate and take-over distance were taken as indices to measure trust. Take-over rate refers to the number of take-over times scaled to the number of emergency events. Take-over distance was defined as the distance from the take-over reaction of the driver to the location of the emergent event. The greater the take-over rate and take-over distance, the more participants chose to drive manually to avoid accidents, and the lower their trust.

\subsection{Procedure}

The experiment was conducted in a lighted room. Participants first completed the CBF-PI-B to measure their personality. After that, they practiced an NDRT, in which they performed an addition task on the hand-held tablet (see Fig. 4 for an illustration). Participants had to enter the result within $3 \mathrm{~s}$. A countdown box was used to display the remaining time. After participants finished the response or did not give a response within $3 \mathrm{~s}$, a new addition task would start. If participants entered a wrong answer, they could press the reset button then enter the right answer. Every 10 trials the program calculated the average accuracy. If a participant's mean accuracy was below $60 \%$, pop-up reminder would inform the participant. Once participants were familiar with the task, they put on the Tobii Pro Glasses 2 and completed the calibration procedure.

The driving started with a $2 \mathrm{~km}$ practice route, enabling participants to learn how to operate the simulator. The practice was the same as the formal experiment except that it was conducted in a different city environment. During practice,

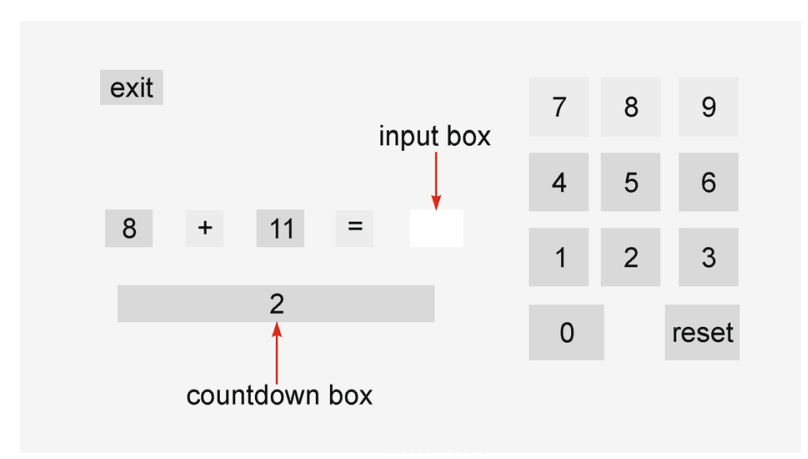

Fig. 4 Addition task (NDRT) interface on the tablet. Participants were told not to press the exit button in the upper left corner 
participants were instructed to learn the operation of NDRT including switching control, braking, throttling and steering the wheel of the simulator, which covered all the operations needed for driving. Participants were also told that the AD system made turns automatically without any manual control in the intersections so they did not need to take over. The practice took about $5 \mathrm{~min}$, and all participants reported that they were familiar and comfortable with the simulator after the practice. After the practice, the participants were informed that although the AD system was safe most of the time, limitations of the L2 AD system meant that it could fail to address emergent events (without informing them of the potential events used in the experiment), leading to accidents. Two accidents would occur if the vehicle was not controlled in time when facing 24 emergent events (i.e., the maximum failure rate was $2 / 24$ ). The two accidents occurred randomly during the experiment. Therefore, when the system was in automated mode, participants had to pay attention to the potential emergent events and take over the system whenever they thought the system might fail to handle the emergent events. Once they considered the situation was safe, they were encouraged (but not mandated) to tune to the $\mathrm{AD}$ mode. To emphasize the importance of driving safety, payment was directly related to accidents, which could occur at most twice if not controlled at all, and each accident would lead to a reduction of 5 RMB (the maximum payment was $50 \mathrm{RMB}$ ). Next, participants watched a video of an accident recorded based on a simulated scene, one which was not used in the formal experiment, to further ensure they understood the possibility of danger.

After the aforementioned preparation session, the formal experiment began (see Fig. 5). The experiment started in AD mode, with a green "Auto" mark in the middle of the steering wheel. Participants were allowed to perform the NDRT when the car was in AD mode. However, the participants were told that they should first guarantee driving safety, and they did not have to perform the NDRT when a take-over was needed. During the experiment, an emergent event, randomly selected from three categories, would occur every 1400-2600 m (corresponding to an average of $110.7 \mathrm{~s} /$ event). Each category occurred 8 times, resulting in a total of 24 events in the whole process. If the participants noticed the

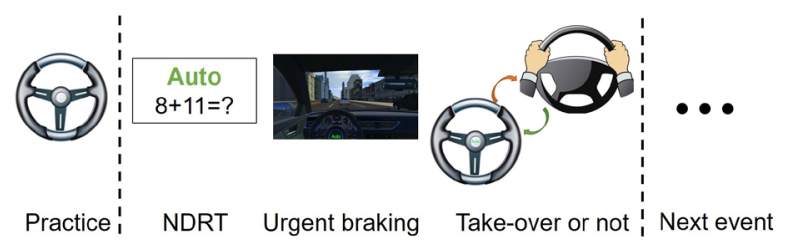

Fig. 5 Schematic illustration of the experimental procedure. Here shows an example of urgent brake emergent event and believed that the AD system could not handle the danger properly, they had to take over the vehicle by hitting the brake pedal to switch the vehicle to manual driving mode (recommended method) or pressing a green button on the simulator. Once the system received a switching signal, the green mark "Auto" switched to red. After passing the emergent event, the participants were encouraged to reset the vehicle to the AD mode by pressing the green button when they believed that the AD system could handle the danger.

The participants had 2-5 min rest after driving $25 \mathrm{~km}$, when the eye-tracker stopped recording. Then the eyetracker was recalibrated to eliminate any possible deviation caused by body movements during the rest. It took about $40 \mathrm{~min}$ to complete the formal experiment and $1 \mathrm{~h}$ to finish the whole experiment (including practice, rest and calibration procedure). After the experiment, the participants completed the trust questionnaire.

\subsection{Analysis}

All the measured variables were at an interval level. A onesample Kolmogorov-Smirnov test was conducted for each measured variable to check whether it conformed to the normal distribution. The scatterplots (Figs. 6, 7, 8) demonstrated that there was a linear relationship between each pair of examined variables. Pearson correlation analyses were conducted between the scores of the CBF-PI-B and that of the AD Trust scale, gaze behavior and driving behavior. Additionally, to control the influence of driving experience on the current results, partial correlation analysis was performed between the scores of the CBF-PI-B and that of AD Trust scale, gaze behavior and driving behavior, with participants' driving experience under control. A significance level of 0.05 was used for statistical tests.

\section{Results}

\subsection{Descriptive Data}

One-sample Kolmogorov-Smirnov tests showed that all the measured variables conformed to normal distributions $(p s>0.20)$.

The descriptive results of the current measured indices are shown in Table 1. The personality scores show that, on average, participants scored highest on the Conscientiousness trait, and lowest on the Neuroticism trait. Participants had a medium level of trust after $\operatorname{AD}(M=3.92)$. During the $\mathrm{AD}$, participants fixated on the AOI 0.57 times per second on average, and the average time they spent monitoring the AOI scaled to the duration of NDRTs was 0.65. Additionally, participants took over the vehicle 9.6 times on average 
a

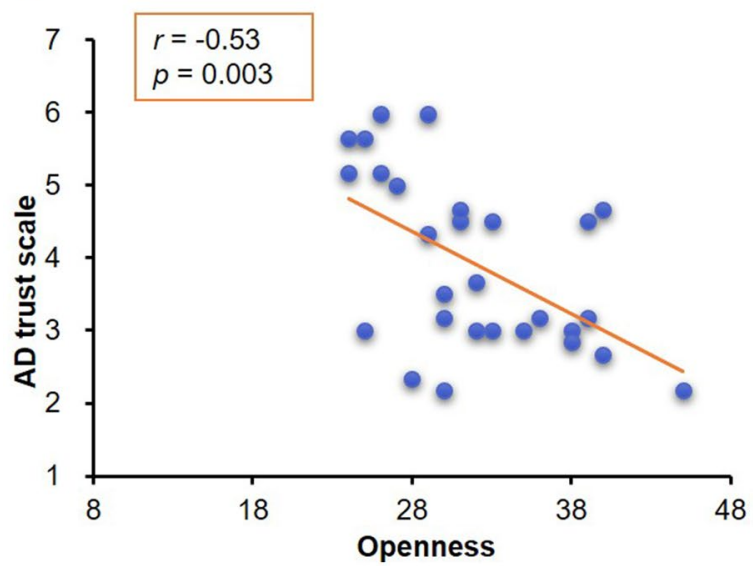

b

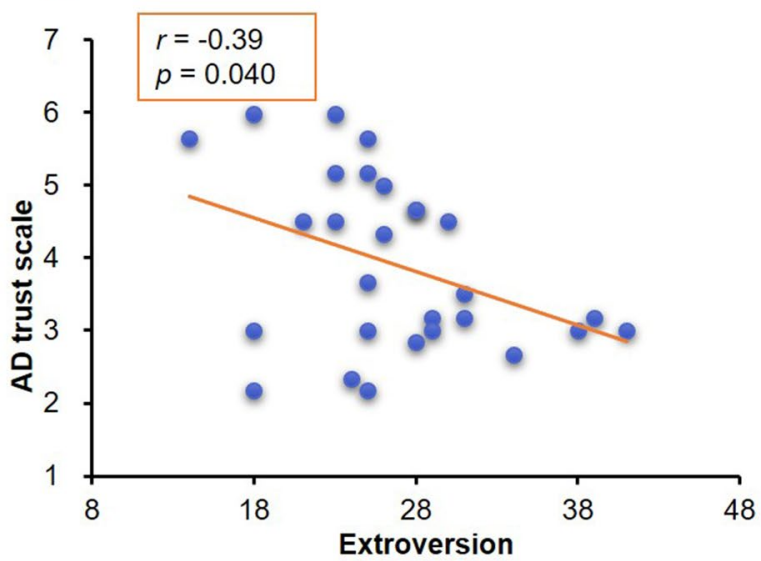

Fig. 6 Pearson correlation results between personality traits and AD trust score: a Openness trait, b Extroversion trait

a

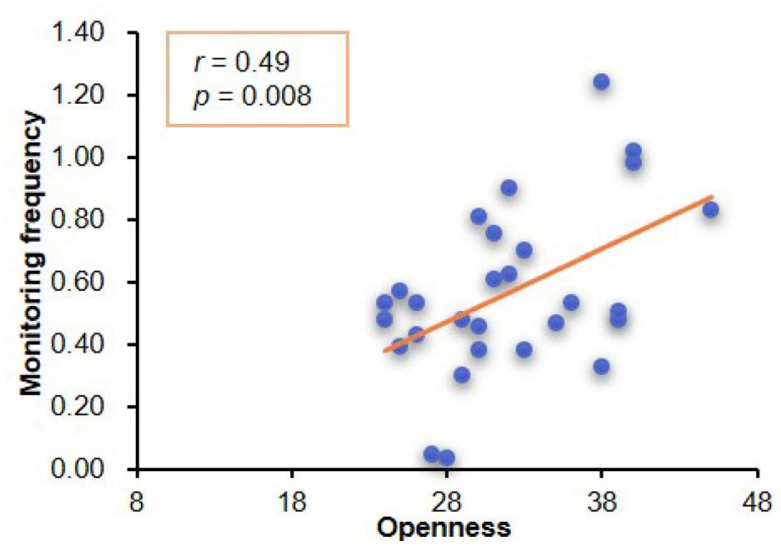

b

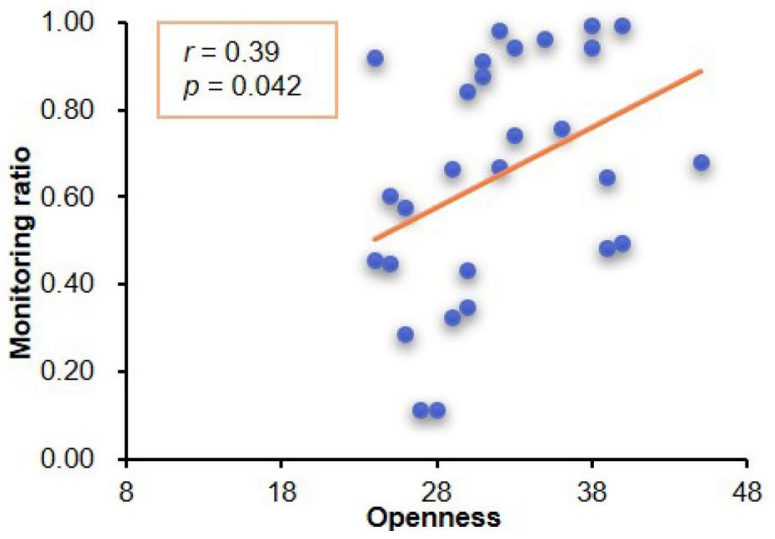

Fig. 7 Pearson correlation results between Openness trait and gaze behavior: a Monitoring frequency, b Monitoring ratio

a

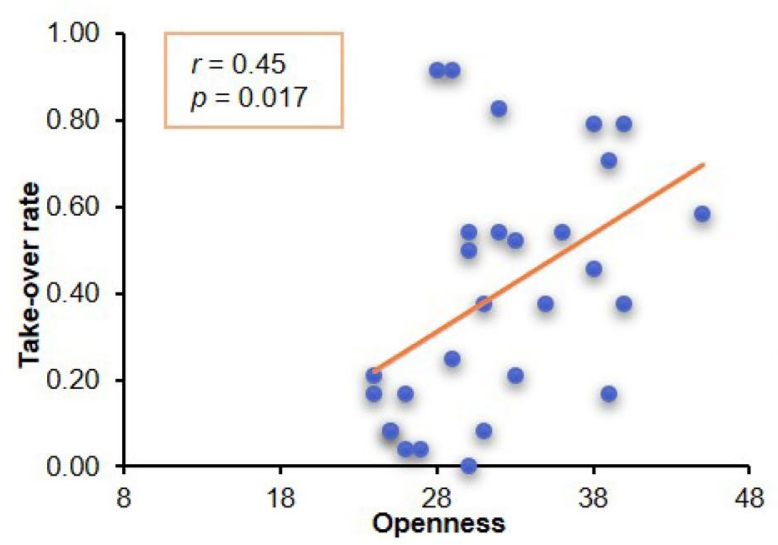

b

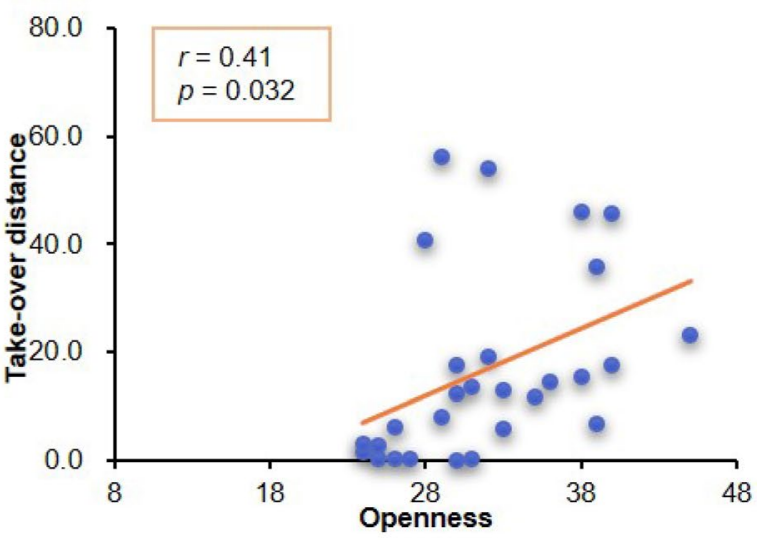

Fig. 8 Pearson correlation results between Openness trait and driving behavior: a Take-over rate and b Take-over distance 
Table 1 Mean and standard deviations (SD) of the measurement indices

\begin{tabular}{lcc}
\hline Measure & Mean & SD \\
\hline Personality & & \\
Neuroticism & 24.14 & 5.51 \\
Conscientiousness & 34.50 & 4.45 \\
Agreeableness & 33.00 & 4.85 \\
Openness & 31.96 & 5.66 \\
Extroversion & 26.61 & 6.34 \\
AD trust score & 3.92 & 1.20 \\
Gaze behavior & & \\
Monitoring frequency & 0.57 & 0.27 \\
Monitoring ratio & 0.65 & 0.27 \\
Driving behavior & & \\
Take-over rate & 0.40 & 0.29 \\
Take-over distance & $16.85 \mathrm{~m}$ & $17.28 \mathrm{~m}$ \\
\hline
\end{tabular}

during the 24 emergent events (take-over rate $=0.40$ ), with an average distance of $16.85 \mathrm{~m}$ from the driver's take-over reaction to the location of the emergent event.

\subsection{Correlation Analysis}

The correlation results are summarized in Table 2.

\subsubsection{Correlation Between Personality Traits and AD Trust Score}

The Pearson correlation analysis revealed a significant negative correlation between Openness and AD trust score $(r=-0.53, p=0.003$, Fig. 6a), as well as a significant negative correlation between Extraversion and AD trust score $(r=-0.39, p=0.040$, Fig. 6b). The Pearson correlations between AD trust and other personality measures were not significant. Moreover, the partial correlation analysis revealed a significant negative correlation between Openness and AD trust score $(r=-0.55, p=0.003)$. The other personality measures failed to reach significance. These results suggest that only the Openness trait has reliable correlation with the $\mathrm{AD}$ trust score: the higher the level of Openness trait, the lower the score of trust scale.

\subsubsection{Correlation Between Personality Traits and Gaze Behavior}

The Pearson correlation analysis revealed a significant positive correlation between Openness and both monitoring frequency $(r=0.49, p=0.008$, Fig. 7a) and monitoring ratio $(r=0.39, p=0.042$, Fig. $7 \mathrm{~b}$ ). The other personality traits showed no significant correlations. Moreover, the partial correlation analysis revealed a similar result: monitoring frequency $(r=0.49, p=0.009)$ and monitoring ratio $(r=0.39$, $p=0.046$ ) were positively correlated with Openness. Other personality traits failed to reach significance. These results suggest that only the Openness trait has correlation with gaze behavior: higher levels of Openness correspond to higher levels of monitoring frequency and ratio to AOI.

\subsubsection{Correlation Between Personality Traits and Driving Behavior}

The Pearson correlation analysis revealed a significant positive correlation between Openness and take-over rate $(r=0.45, p=0.017$, Fig. $8 \mathrm{a})$, and take-over distance $(r=0.41, p=0.032$, Fig. 8 b). The other personality measures did not reach significance. Similarly, the partial correlation analysis revealed a significant positive correlation between Openness and take-over rate $(r=0.46, p=0.017)$ and takeover distance $(r=0.42, p=0.031)$. These results suggest that only Openness trait has correlation with take-over behavior: higher levels of Openness correspond to both more frequent and earlier take-over behavior.
Table 2 Results of correlation between the personality traits and each trust indices

\begin{tabular}{llllll}
\hline Personality & Trust score & Take-over distance & Take-over rate & $\begin{array}{l}\text { Monitoring } \\
\text { frequency }\end{array}$ & Monitoring ratio \\
\hline Neuroticism & 0.15 & 0.01 & -0.08 & -0.10 & -0.19 \\
& $(0.16)$ & $(0.01)$ & $(-0.09)$ & $(-0.10)$ & $(-0.19)$ \\
Conscientiousness & -0.24 & 0.18 & 0.30 & 0.21 & 0.34 \\
& $(-0.32)$ & $(0.26)$ & $(0.38)$ & $(0.23)$ & $(0.34)$ \\
Agreeableness & -0.01 & 0.07 & 0.16 & 0.21 & 0.17 \\
& $(-0.04)$ & $(0.10)$ & $(0.19)$ & $(0.21)$ & $(0.16)$ \\
Openness & $-0.53^{* *}$ & $0.41^{*}$ & $0.45^{*}$ & $0.49^{* *}$ & $0.39^{*}$ \\
& $\left(-0.55^{* *}\right)$ & $\left(0.42^{*}\right)$ & $\left(0.46^{*}\right)$ & $\left(0.49^{* *}\right)$ & $\left(0.39^{*}\right)$ \\
Extroversion & $-0.39^{*}$ & -0.01 & 0.09 & 0.27 & 0.21 \\
& $(-0.35)$ & $(-0.07)$ & $(0.03)$ & $(0.26)$ & $(0.23)$ \\
\hline
\end{tabular}

The results in parentheses were results of partial correlation analysis $* p<0.05, * * p<0.01$ 


\section{General Discussion}

The current study directly investigated the relationship between driver personality and driver trust in the $\mathrm{L} 2 \mathrm{AD}$ system. Participants were required to experience a $50 \mathrm{~km}$ L2 AD driving route in a 6-DOF simulator, during which their gaze, driving behavior as well as their subjective $\mathrm{AD}$ trust scores after the AD experience were recorded. Three distinct measures consistently revealed a significant negative correlation between Openness and driver trust in the AD: Participants with higher Openness traits tended to have less trust in the AD system. However, no such relationships were found for the other four personality traits.

Due to the limitations of the correlational study, specific reasons explaining the observed negative correlation between Openness and AD trust cannot be provided. One reason might be rooted in the incompatibility between the current setting of the AD system and the inner personality requirements of drivers who score highly on Openness. Specifically, high-Openness individuals are inclined to seek "intellectually challenging activities" to meet their requirement for enjoyment of novelty and curiosity $[26,31]$. However, the setting of the AD system (at least the current simulator) disabled drivers from performing intellectually challenging activities. In the experimental setting, the participants could only perform an NDRT and take over the system in certain emergent situations. The NDRT was a very simple digit addition task for the participants, who are all college students, because even fourth-grade primary school students can complete it easily. Moreover, although for most participants, this experiment was their first exposure to $A D$, the interface of the simulator was similar to a manual car, and all participants were given sufficient practice. Taking these facts into consideration, it is suggested that the current task setting was not intellectually challenging to the participants. In other words, there was no leeway for drivers to investigate the system or to experience novelty or curiosity. Future empirical studies may consider measuring driver preference for novelty and curiosity to verify this explanation.

The current study contributes new evidence supporting the view that user personality has a tight relation with trust in automation, by focusing on the L2 AD system. In contrast to [24], which found that both Agreeableness and Conscientiousness traits predict one's initial trust in automation, a significant negative correlation with the trait of Openness was found. However, the two studies are not necessarily contradictory to each other. Chien et al. [24] examined propensity to trust automation in general (i.e., participants did not interact with a specific system). Instead, the current study examined trust in a specific system, both while participants interacted with the system (ongoing trust; measured by gaze behavior and driving behavior) and after their interactions with the AD system (post trust; measured by the Trust scale). Taking the study of Chien et al. [24] and the current study together, it seems that personality affects trust propensity and ongoing and post-trust in automation. This relationship has been largely neglected in previous individual difference studies but has important practical implications (see the following discussion). Meanwhile, as the first study of this type, the current study only examined ongoing/post-trust in an AD system. Future study should consider the relation between personality and trust propensity in an AD system.

In addition, within the same type of measure, significant correlations were found: take-over distance was positively correlated with take-over rate (Pearson $r=0.94, p<0.01$ ); monitoring frequency was positively correlated with monitoring rate (Pearson $r=0.58, p<0.01$ ). Between different measures, only a significant correlation between the score of trust scale and take-over rate was found (Pearson $r=-0.44$, $p<0.05)$; the other relations were not significant. It is suggested that the lack of correlations between different measures is because they reflect different aspects/sources of trust. However, this remains to be verified.

Trust has been considered a key determinant of the willingness of humans to rely on automated systems [1, 11]. Exploring factors affecting AD trust has significant theoretical implications. Indeed, the current study shed light on trust calibration. To be specific, since an appropriate level of trust in the $\mathrm{AD}$ system is critical for safe driving, it is important to understand the potential factors affecting trust calibration, which means dynamically changing the level of trust in the automation in accordance with system performance. The current study, reveals a crucial psychological factor (i.e., Openness trait) that affects trust in the AD system. This finding implies that trust calibration needs to account for the personality trait of Openness, to avoid excessive trust of the $\mathrm{AD}$ system by low-Openness individuals or too little trust of the AD system by high-Openness individuals. An optimized HMI could be achieved, for instance, by modifying AD system's reaction strategy to emergent events or offering different warning systems to different types of drivers.

Finally, several limitations should be acknowledged. First, since the current study was conducted with an AD simulator, the operational and working environment of the simulator is quite different from real AD systems which contains multiple state-of-the-art displays and control elements; hence, it might meet the novelty and curiosity requirements of users (at least at the very beginning). Moreover, participants might believe that accidents could cause no real harm, which would influence their take-over behavior. Although a penalty was introduced to minimize such false belief, a driver might take over the vehicle as soon as an emergent event occurred if he 
or she cared a lot about remuneration. Therefore, additional studies are needed to verify the current findings on real AD systems. Second, participants were told to press the button or hit the brake pedal when they faced emergent events in the study. However, not everyone's first reaction is to hit the brake pedal; some people are used to controlling the steering wheel first. The take-over methods could be optimized in further studies. Third, additional studies are needed to examine the potential factors leading to the current finding. For instance, it is possible that participants' unmet need for intellectually challenging activities in the simulator result in annoyance or boredom, which then serves in a mediating role to affect trust. Additionally, because AD is a new technology, people could differ in how open they are to new technologies. It is also possible that a person's attitude to new technology affects AD trust. Therefore, future study could test those factors by measuring participants' annoyance or boredom during AD and their openness to new technology [40], following Rogers' Innovation Diffusion Theory. Finally, the participants in the current study were all college students, creating a young distribution (23.3 years old \pm 1.4 ). Moreover, driver license years can vary with real age. Therefore, the current finding may be limited to young college students with limited driving experience. Future study needs to examine whether the current findings generalize to all age groups and whether the current finding is moderated by driving experience.

\section{Conclusions}

In three distinct measures, a significant correlation between Openness and driver trust in the AD simulator is found: Participants with higher Openness trait tend to have less trust in the AD system. However, such correlation does not hold for the other four personality traits. The current study suggests that driver personality has an impact on the trust in AD.

Acknowledgements This research is supported by HIRPO2017150309. We thank Yaqi Liu for gorgeous help during the project.

\section{Compliance with Ethical Standards}

Conflict of interest The authors declare that they have no conflict of interest.

Open Access This article is licensed under a Creative Commons Attribution 4.0 International License, which permits use, sharing, adaptation, distribution and reproduction in any medium or format, as long as you give appropriate credit to the original author(s) and the source, provide a link to the Creative Commons licence, and indicate if changes were made. The images or other third party material in this article are included in the article's Creative Commons licence, unless indicated otherwise in a credit line to the material. If material is not included in the article's Creative Commons licence and your intended use is not permitted by statutory regulation or exceeds the permitted use, you will need to obtain permission directly from the copyright holder. To view a copy of this licence, visit http://creativecommons.org/licenses/by/4.0/.

\section{References}

1. Lee, J.D., See, K.A.: Trust in automation: designing for appropriate reliance. Hum. Factors 46(1), 50-80 (2004)

2. Lee, J., Moray, N.: Trust, control strategies and allocation of function in human machine systems. Ergonomics 35(10), 1243-1270 (1992)

3. Lewandowsky, S., Mundy, M., Tan, G.: The dynamics of trust: comparing humans to automation. J Exp. Psychol. Appl. 6(2), 104-123 (2000)

4. Muir, B.M.: Trust in automation: part I. Theoretical issues in the study of trust and human intervention in automated systems. Ergonomics 37(11), 1905-1922 (1994)

5. Muir, B.M., Moray, N.: Trust in automation: part II. Experimental studies of trust and human intervention in a process control simulation. Ergonomics 39(3), 429-460 (1996)

6. Parasuraman, R., Riley, V.: Humans and automation: use, misuse, disuse, and abuse. Hum. Factors 39(2), 230-253 (1997)

7. Riley, V.: Operator reliance on automation: theory and data. In: Parasumaran, R., Mouloua, M. (eds.) Automation and human performance: theory and applications, pp. 19-35. Erlbaum, Hillsdale (1996)

8. Kelleher, K.: Man arrested for drunk driving after officers found him asleep in tesla running in autopilot mode. Fortune. http:// fortune.com/2018/11/30/man-arrested-drunk-driving-aslee p-tesla-autopilot-mode/ (2019). Accessed 10 May 2019

9. Morris, Z.D.: Tesla driver, passed out drunk, tells highway patrol autopilot was in charge. Fortune. http://fortu ne.com/2018/01/22/tesla-drunk-driver-autopilot/ (2019). Accessed 10 May 2019

10. National transportation safety board.: preliminary report: crash and post-crash fire of electric-powered passenger vehicle. NTSB. https://www.ntsb.gov/investigations/AccidentReports /Pages/HWY18FH011-preliminary.aspx (2019). Accessed 10 May 2019

11. Hoff, K.A., Bashir, M.: Trust in automation: integrating empirical evidence on factors that influence trust. Hum. Factors 57(3), 407-434 (2015)

12. Gold, C., Körber, M., Hohenberger, C., et al.: Trust in automation-before and after the experience of take-over scenarios in a highly automated vehicle. Procedia Manuf. 3, 3025-3032 (2015)

13. Molnar, L.J., Ryan, L.H., Pradhan, A.K., et al.: Understanding trust and acceptance of automated vehicles: an exploratory simulator study of transfer of control between automated and manual driving. Transp. Res. Part F Traffic Psychol. Behav. 58, 319-328 (2018)

14. Helldin, T., Falkman, G., Riveiro, M., et al.: Presenting system uncertainty in automotive UIs for supporting trust calibration in autonomous driving. In: Proceedings of the 5th International Conference on Automotive User Interfaces and Interactive Vehicular Applications, ACM, Eindhoven, 28-30 Oct 2013

15. Verberne, F.M., Ham, J., Midden, C.J.: Trust in smart systems: sharing driving goals and giving information to increase trustworthiness and acceptability of smart systems in cars. Hum. Factors 54(5), 799-810 (2012)

16. Koo, J., Kwac, J., Ju, W., et al.: Why did my car just do that? Explaining semi-autonomous driving actions to improve driver understanding, trust, and performance. Int. J. Interact. Des. Manuf. 9(4), 269-275 (2015) 
17. Waytz, A., Heafner, J., Epley, N.: The mind in the machine: anthropomorphism increases trust in an autonomous vehicle. J. Exp. Soc. Psychol. 52, 113-117 (2014)

18. Zihsler, J., Hock, P., Walch, M., et al.: Carvatar: increasing trust in highly-automated driving through social cues. In: Adjunct Proceedings of the 8th International Conference on Automotive User Interfaces and Interactive Vehicular Applications, ACM, Ann Arbor, 24-26 Oct 2016

19. Hergeth, S., Lorenz, L., Vilimek, R., et al.: Keep your scanners peeled: gaze behavior as a measure of automation trust during highly automated driving. Hum. Factors 58(3), 509-519 (2016)

20. Payre, W., Cestac, J., Delhomme, P.: Fully automated driving: impact of trust and practice on manual control recovery. Hum. Factors 58(2), 229-241 (2016)

21. Szalma, J.L., Taylor, G.S.: Individual differences in response to automation: the five factor model of personality. J. Exp. Psychol. Appl. 17(17), 71-96 (2011)

22. Rödel, C., Stadler, S., Meschtscherjakov, A., et al.: Towards autonomous cars: the effect of autonomy levels on acceptance and user experience. In: Proceedings of the 6th International Conference on Automotive User Interfaces and Interactive Vehicular Applications, ACM, Seattle, 17-19 Sept 2014

23. Schaefer, K.E., Scribner, D.R.: Individual differences, trust, and vehicle autonomy: a pilot study. Proc. Hum. Factors Ergon. Soc. Annu. Meet. 59(1), 786-790 (2015)

24. Chien, S.Y., Lewis, M., Sycara, K., et al.: Relation between trust attitudes toward automation, Hofstede's cultural dimensions, and big five personality traits. Proc. Hum. Factors Ergon. Soc. Annu. Meet. 60, 841-845 (2016)

25. Mcbride, M., Carter, L., Ntuen, C.: The impact of personality on nurses' bias towards automated decision aid acceptance. Int. J. Inf. Syst. Change Manag. 6(6), 132-146 (2012)

26. Costa, P.T., McCrae, R.R.: Neo personality inventory-revised (NEO PI-R). Psychological Assessment Resources, Odessa (1992)

27. Matthews, G., Deary, I.J., Whiteman, M.C.: Personality traits, 2nd edn. Cambridge University Press, Cambridge (2003)

28. Matthews, G., Joyner, L., Gilliland, K., et al.: Validation of a comprehensive stress state questionnaire: towards a state "big three"? In: Mervielde, I., Deary, I.J., De Fruyt, F., et al. (eds.) Personality psychology in Europe, vol. 7, pp. 335-350. Tilburg University Press, Tilburg (1999)

29. Matthews, G., Campbell, S.E.: Task-induced stress and individual differences in coping. Proc. Hum. Factors Ergon. Soc. 42(11), 821-825 (1998)

30. Penley, J.A., Tomaka, J.: Associations among the big five, emotional responses, and coping with acute stress. Pers. Individ. Differ. 32(7), 1215-1228 (2002)

31. Ackerman, P.L., Heggestad, E.D.: Intelligence, personality, and interests: evidence for overlapping traits. Psychol. Bull. 121(2), 219-245 (1997)

32. SAE On-Road Automated Vehicle Standards Committee: Taxonomy and definitions for terms related to driving automation systems for on-road motor vehicles. SAE International, Warrendale (2018)

33. Wang, M., Dai, X., Yao, S.: Development of Chinese big five inventory (CBF-PI) I: theoretical framework and reliability analysis. Chin. J. Clin. Psychol. 18(5), 545-548 (2010)

34. Wang, M., Dai, X., Yao, S.: Development of Chinese big five inventory (CBF-PI) II: validity analysis. Chin. J. Clin. Psychol. 18(6), 687-690 (2010)

35. Wang, M., Dai, X., Yao, S.: Development of Chinese big five inventory (CBF-PI) III: psychometric properties of CBF-PI brief version. Chin. J. Clin. Psychol. 19(4), 454-457 (2011)

36. Jian, J.Y., Bisantz, A.M., Drury, C.G.: Foundations for an empirically determined scale of trust in automated systems. Int. J. Cogn. Ergon. 4(1), 53-71 (2000)

37. Ahmad, A., Anawar, H.N.: Association between femininity and women voters political trust in Pakistan. J. Hum. Behav. Soc. Environ. 28(5), 664-672 (2018)

38. McCann, S.J.: Big five personality and residential mobility: a state-level analysis of the USA. J. Soc. Psychol. 155(3), 274-291 (2015)

39. Nave, G., Minxha, J., Greenberg, D.M., et al.: Musical preferences predict personality: evidence from active listening and facebook likes. Psychol. Sci. 29(7), 1145-1158 (2018)

40. Moore, G.C., Benbasat, I.: Development of an instrument to measure the perceptions of adopting an information technology innovation. Inf. Syst. Res. 2(3), 192-222 (1991) 